\title{
A women's perspective on premenstrual syndrome: a qualitative interview study
}

Marijke Labots-Vogelesang ( $\nabla$ marijke.labots-vogelesang@radboudumc.nl )

Radboud University Medical Center https://orcid.org/0000-0002-2369-5998

\section{Rachel Kooiman Andringa}

Radboud University Medical Center

Doreth A M Teunissen

Radboud University Medical Center

Antoine LM Lagro Janssen

Radboud University Medical Center

Research article

Keywords: Premenstrual Syndrome, Premenstrual Disorders

Posted Date: July 3rd, 2019

DOI: https://doi.org/10.21203/rs.2.10868/v1

License: (c) (1) This work is licensed under a Creative Commons Attribution 4.0 International License. Read Full License 


\section{Abstract}

BMWH-D-18-00422 A women's perspective on premenstrual syndrome: a qualitative interview study Abstract Background: Women who present with Premenstrual Syndrome (PMS) to healthcare professionals experience a lot of problems on their psychosocial functioning. Little is known however about the influence of PMS on the daily life of women in the general population. The aim of the present study is to improve our understanding of the perspective of women with PMS in the general population. Methods: We recruited women with PMS via local newspapers and social media. We performed semistructured interviews. Results: We interviewed twenty women between 27 and 49 years of age. Physical and in particular psychological symptoms of PMS have a strong influence on the quality of life of women. Three themes emerged from our analysis. Women mentioned disturbance in their preferred roles of being a good mother and wife; experienced PMS as a life controlling condition and they used active and passive coping strategies with different expectations of healthcare. Conclusion: The symptoms of PMS affect women at the level of their social role of femininity. These women are in need of acknowledgement and support as well from family and friends as from healthcare providers. Word count: 3772 (abstract 211) Keywords: Premenstrual Syndrome, Women's Health, Social Role, Femininity, Interview, Coping, Acknowledgement

\section{Background}

Premenstrual Syndrome or PMS is characterized by physical, mental and behavioural symptoms during the luteal phase of almost every menstrual cycle, the most common being irritability, mood swings, tense or depressed mood, breast tenderness and bloating [1-5]. PMS is characterized by clear functional impairment, although many women of reproductive age do experience mild premenstrual symptoms and a certain degree of discomfort is to be considered as mainly physiological. Women with PMS often experience considerable burden in their daily psychosocial functioning such that it is, due to its chronicity, being compared to a depressive disorder [4-6]. Although the prevalence of PMS in conformity with the DSM-4 criteria among European women is estimated between 4 and $8 \%[7,8]$. This condition amongst women received only little acknowledgement. Several treatment strategies have been suggested for PMS, but cognitive behavioural therapy, selective serotonin reuptake inhibitors and contraceptives are supported by only moderate evidence $[9,10]$.

Oddly, the perspective of women with PMS in the general population has scarcely been studied. It is not known to what degree these women are encumbered by PMS, with what consequences for their daily lives, how they cope with the distress and what they might expect from their doctor $[11,12]$. For healthcare professionals it is important to know what women experience as invalidating or bothersome in order to optimize care for these women specifically. To our knowledge there is no consensus guideline for primary care to approach women with PMS. The existing consensus, developed by experts represented in the ISPMD, is based on specialist research [13]. We hypothesized that for this purpose, the women's perspective should be taken into account. Therefore, in this qualitative study we investigated which 
symptoms or complaints are considered most disabling and why, what cognitions women have about the cause of PMS and how these affect their help-seeking behaviour.

\section{Methods}

Study design

Using a qualitative study design, we performed in-depth, semi-structured interviews to examine personal experiences, cognitions, and expectations of women in general population reporting symptoms of PMS.

\section{Study population}

Women experiencing premenstrual symptoms were invited to participate in the study through local newspapers and social media. We rephrased the DSM- 5 criteria in lay terms. Women could register for an intake by telephone. During the intake by phone, an experienced researcher (RK, ML) took the time to check by listening and asking whether the premenstrual symptoms mentioned by the respondent fitted into the DSM-5 criteria for PMS. Only women whose symptoms met the identified criteria were admitted to the study. Table 1 (see Supplemental Materials) lists the inclusion criteria. To be sure of the cyclical nature we verified when these symptoms started and explicitly on which day of menstruation these disappeared. With these criteria we included, from thirty respondents, twenty-five women. After we had recruited ten participants, ten more women were included by purposive sampling to reach as much variation as possible by asking them about their age, education level, employment and ethnicity. Five respondents did not respond to the invitation or were short of time. After conducting twenty interviews, by telephone or face-to-face [14], we ceased recruitment of new participants as we had reached saturation, which is further detailed below.

\section{Data collection}

Between March 2017 and May 2017, two researchers from the Department of Primary and Community Care and Gender \& Women's Health (ML, RK) at Radboud University Medical Center conducted in-depth, semi-structured interviews using an interview guide. The interview guide has been compiled on the basis of literature and advice of an expert committee (ML, TT, ALJ) [Box 1]. After five interviews, we added to the third theme of the interview guide two questions about what women believed to be the origin and cause of their premenstrual symptoms and what it could worsen.

Prior to the interview the participants gave their written consent. After completing a general questionnaire about background characteristics the participants were interviewed about their symptoms, cognitions, and experiences with regard to PMS.

\section{Data analysis}

Interviews were recorded on audio equipment with the participants' consent and were transcribed verbatim. Two researchers (ML, RK) independently analysed the transcripts so as to limit the researchers' 
interpretation and increase reliability. We used Atlas.ti, version 7.1.5 for open thematic coding.

The codes were compared and discussed after every five interviews to reach consensus. After twenty interviews, we stopped inclusion as no more new codes appeared in the last two interviews. We discussed the resulting list of codes, which we clustered into categories and discussed with the supervisor's committee. The categories were grouped into three themes. We supported our interpretation of the findings with quotations. We provided numbers to specify if results were raised by few (1-4), some (5-9), many (10-15), or most (16-19) participants. We applied the COREQ criteria for reporting qualitative research [15].

\section{Results}

\section{Participants}

We interviewed twenty women in the age of 27 to 49 years [Table 2] (see Supplemental Materials). All women in general considered it important that their symptoms were recognized by healthcare professionals. They found it helpful and reassuring to label themselves with PMS, and to know that how they felt or behaved was not because of them but because of PMS.

Most women reported that the psychological symptoms were considerably more troublesome and limiting for their daily lives than the physical symptoms, which is underlined by a few women whom reported to even have had thoughts about ending their life. In addition to this general opinion we categorized the important and relevant views emerging from our analysis of the data, for an example see Table 3 (see Supplemental Materials). We categorized the views in three themes, namely: the disturbance in preferred feminine roles of being a good mother and wife, meaning that the women felt differently during the premenstrual period, as if they were no longer themselves, as a natural woman; PMS as a lifecontrolling condition, meaning that the influence of PMS was great in all areas of life; and differences in coping strategies. These themes have been detailed below.

\section{Disturbance in preferred feminine roles}

Although some women said that PMS was part of being normal, as a natural woman, many women described themselves as insecure about their womanhood, their appearance, or their feminine social behaviour. Women experienced tensions in their relationships as their partners and children had to deal with someone who was alternating in her behaviour and possibilities. PMS therefore affected the way they were mother or partners.

These women seemed to condemn themselves and noticed that their symptoms made them fail to meet social roles of femininity they felt were expected of them: they lacked patience, attention or kindness; they were aggressive or acted like nasty women (original: bitches, in Dutch often used to indicate nasty female behaviour). Some women felt guilty because they were unable to fulfil their wished role as mum and wife in the PMS weeks. 
"And yes, sometimes I feel very guilty towards my children and also towards my husband. [...] I just want to be a normal family member and just a fun mom for my children."

P20 32yr

\section{A life-controlling condition}

Many women considered the PMS symptoms life-controlling: in the choices they made, in the contact with the people they met, the opportunities they faced were influenced by PMS. During the weeks without symptoms, these women were preparing for or worrying about the next PMS phase.

"I'm actually always thinking about it. And when I feel good, I'm already preoccupied with it, like: 'Oh, I hope I won't feel bad again."'

P20 32yr

Almost every woman mentioned that she didn't feel normal during their premenstrual phase. Some women found it difficult to distinguish whether their behaviour was their own or due to PMS.

"No, no. It is indeed real, like being a different person, you know".

P19 28yr

After diminishing of symptoms following the initiation of menstruation, these women felt as if they were becoming their normal selves again. PMS changed their sense of being normal and emphasized that their feeling with PMS was abnormal.

"You feel like something else takes control.[...] And when it's over, I say: 'it's away'. In this way I try to put it outside myself...."

P16 44yr

Some women described a decline in their self-esteem and self-confidence when the PMS phase started.

"That's the worst thing [...] I'm an experienced person and my work went well, but suddenly I started to ask questions [...] to doubt things."

P3 41yr

These alternating periods of feeling good, but also fear of misunderstanding and misjudgements, impeded their social activities.

In addition, many women indicated that their PMS influenced their working activities as well, missing concentration, overview, and efficiency during their PMS phase. Half the women decided to work fewer 
hours or to discontinue work at all because of PMS. Some women said they often called in sick during PMS any worse.

"I stopped my work in February..., called in sick [...], because it all doesn't work any longer [...]; always worked, never been ill."

P11 46yr

PMS seemed to have such an impact that along with depressive and negative feelings, even thoughts about suicide were not exceptional among the interviewees. Some women mentioned recurrent thoughts about ending their life or not valuing life because of the changed lives they led. Few women had attempted to commit suicide at least once.

"And so at some time you [...] also got the tendency to step out of life."

P4 49yr

Differences in coping strategies

Approaches for coping with the symptoms of PMS could roughly be grouped into a coping style with an active approach and a more passive coping style with acquiescing, looking away and avoidance attitude.

Active coping strategy

Most women considered PMS caused by biological mechanisms and used an active, somatic approach and preferred a specialized doctor or clinic. They expressed their need for diagnostic tools to determine whether their hormones were causing the symptoms. More than half these women had disappointing experiences with the medical professionals, gynaecologists, or psychiatrists they consulted, feeling they were not being taken seriously or that the professionals showed little symptom recognition and even less knowledge of proper treatment.

"If there's a specialized department for women with such complaints, so they're really being taken seriously and so you won't be told: 'oh well, that's just part of it."'

P6 27yr

Women reported to feel like they 'needed to be their own doctors'. Many women used different ways to improve their situation: they sought distraction, searched for new information online, saw several doctors or therapists, and tried numerous medicines or therapies. Some women set priorities in their life-styles, or planned their schedule differently in PMS weeks. Others attempted to reduce the impact of PMS by alleviating the problem directly.

"It's one of the characteristics of PMS: if you're having symptoms, you're just looking for solutions, solutions, [...] are still searching online at two o'clock at night: 'maybe I might do this or maybe this will 
work?"'

P8 34yr

Many of these women reported that they actively searched for peers and found it helpful to share experiences or get recognition from others, which made them feel less alone. However, others also mentioned that the despondency or hopelessness of peers could have a worsening influence on their wellbeing.

"It's two-sided [...] you're not the only one. [...] I get a lot of knowledge from them. On the other hand, though, there's a lot of women with negative stories, and I'd rather not be confronted with those if I'm in a good mood...."

P13 36yr

Passive coping strategy

A smaller part of the women coped by just trying to accept the fact they had PMS. They were putting up with it and added self-mockery or tried to laugh the symptoms away, applying behaviour that reduced PMS distress by distancing themselves from the problem. These women were more likely to be surprised by the new PMS-phase; some said they repeatedly underestimated their complaints when PMS was over and were overwhelmed every new period of symptoms.

"But just to think of these complaints: 'Okay, let it be,' instead of: 'I don't want this. Here we go again.'[..].And some level of acceptance helps. So to let it be rather than to fight it."

P12 41yr

"And [...] you can no longer turn to a doctor, because [...] they had no answer to that either. [...] Yes, around my period I am slightly adjusting my lifestyle and I separate myself somewhat."

$\mathrm{P} 4,49 \mathrm{yr}$

'Some women considered it important for their healthcare professional to have a holistic view on PMS and to address PMS in a more integrated way than just physically. Nevertheless, they were open to a variety of treatments: medication, psychotherapy, alternative counselling or group conversations.

"If I don't mention my complaints, I don't have to expect, that she (my GP) will do anything for me either. But if any treatment is possible, I think, I would like to use it."

$\mathrm{P} 1,31 \mathrm{yr}$

\section{Discussion And Conclusion}


Although the existence of premenstrual symptoms and symptoms as PMS is well known, the perspective of women with PMS regarding its burden and its cause thus far received only minor attention. The most important finding of our qualitative study among twenty women identified in the general population is the disruption between the women's own definition with regard to their good phases without PMS and their bad phases with PMS. A majority reports the disturbing of their normal lives at the beginning of PMS, as the most disabling consequence. Women describe that they felt to have two separate identities, one belonging to life without and one to life with symptoms. This is in line with findings of studies among patients with chronic or recurrent illnesses who face identity changes because of their illnesses [16]. The impairment of normal social life, the feeling of not being themselves during PMS phases and the inability to organize their lives to fulfil their role as mother, wife, as they expect from themselves. PMS disturbs their wished role and affects their femininity, as being no longer 'normal', a natural woman [17]. We found no indication of possible disruptive circumstances in the relational sphere due to PMS. On the contrary, women felt more guilty towards their family [18].

All selected women in this study experienced a great burden of PMS, with at least one negative mood symptom. Some studies also show positive symptoms. Including questions about positive symptoms into a questionnaire will increase the reporting of positive symptoms [19]. However, we reported on women with negative premenstrual experiences, as these are the women who usually go to healthcare for help and in whose experiences we were interested.

Current coping literature often distinguishes approach/active coping style and avoidance/passive coping style, which are also evident in our study $[20,21]$. Many women considered PMS to be a major problem. Women with a more active coping strategy stated to be more in need of a specialized approach and searched for all possible treatments available to relieve their symptoms, also depending on the women's cognitions about causality. They are in need of acknowledgement, diagnostics, and somatically focused treatment [21]. Some other women, with a more passive coping strategy, considered their symptoms as a natural part of being a woman and tried to learn dealing with them. These women stated to be in need of a more general or integrated approach. However, they also expect recognition, social support and a psychosocial view.

In contrast to the results from our study, most participants from a more selected population showed a more passive coping strategy. But aspects as dealing with self-regulation of premenstrual distress seem to fit more closely with our theme of passive coping strategy [22].

An interesting way of thinking is considering not only the style of coping, but also the symptoms of PMS itself, as a way of coping. The symptoms appropriate to PMS, as varied as these may be, are then the result of (premenstrual) distress evoked by premenstrual changes [22].

Nevertheless, all women with PMS expect recognition. Unrecognition of the different needs of women depending on their coping styles may give rise to miscommunication, discontent, and tension in the doctor-patient relationship. The challenge for healthcare professionals lies in exploring the patients' views and expectations and the context and cognitions that are related to them. In this regard, it is of great 
importance for both doctors and patients to pursue an open communication style when dealing with PMS. This is all the more important due to the fact that some women considered or even attempted suicide because of the burden of PMS [4].

We favour that PMS should rather be seen in the light of a biopsychosocial model, which focuses not only on biomedical aspects but also on psychological and social factors that are crucial to disease and to the healing process [23].

The strengths of our study include our great variability in age, parity, employment in our study population and reaching saturation in our data collection. A major pitfall of a qualitative approach is the interference of subjectivity, which we precluded by independently encoding and analysing all interviews and codes with two researchers and by structural and objective consultation of the supervisors' committee.

A limitation of our study is that the number of women with ethnic backgrounds other than Dutch is low. It is difficult to reach women from other cultures as they are less likely to respond to advertisements due to cultural restrictions in talking about premenstrual problems. Some recall bias cannot be ruled out as, during the interview, the women reflected on their symptoms from their experience. For women who have been suffering from monthly premenstrual problems for a long time, thickening of their symptoms is less likely. However, the symptoms may have diminished or changed in possibly premenopausal women as well.

Selection bias, lastly, could have occurred by including women who were willing to participate in a study and eager to tell their stories and who, therefore, are more likely to experience a greater burden of PMS.

\section{Conclusion}

This study shows that precisely the mental symptoms of PMS strongly interfere with the self-perception and feminine identity of women with PMS. These women may experience disturbance in their role of femininity, being a natural woman, consider PMS as a life-controlling condition and use active as well as passive coping strategies related to their cognitions about PMS. Biomedical aspects as well as psychological and social aspect should be acknowledged. Individual patients require understanding by their social support system and by healthcare providers.

\section{Abbreviations}

PMS: Premenstrual Syndrome

GP: general practitioner

COREQ: criteria for reporting qualitative research

WMO: Medical Research Involving Human Subject Acts

ISPMD: International Society for Premenstrual Disorders 


\section{Declarations}

Ethics approval and consent to participate

The Radboudumc Research Ethics Committee ruled that it does not fall within the remit of the Medical Research Involving Human Subject Acts (WMO) because of the non-invasive character of this interview study (Case number 2017-3139).

Consent to publish:

Participants were asked to give their written informed consent prior to the interview.

Availability of data and material

The datasets generated and analysed during the current study are not publicly available due reasons of confidentiality, but are available from the corresponding author on reasonable request.

Authorship statement

$\mathrm{ML}, \mathrm{RK}, \mathrm{TT}$ an ALJ fulfilled the criteria for authorship and contributed to this paper. All authors except RK were involved in the conception and design of the study. ML, RK, TT an ALJ contributed substantial to the acquisition, analysis and interpretation of data, revising the article and the final approval of the paper. ML is the principle researcher and responsible for the data collection.

Competing interests

All authors indicated no potential competing interest.

Funding

This work was supported by a small grant of the Dutch Society of Female Doctors (2017, VNVA).

Acknowledgements

The authors thank all the women who participated in the study. We thank Chrissy de Bree for her assistance in the coding process of the data.

\section{References}

[1] Yonkers KA, O'Brien PM, Eriksson E. Premenstrual syndrome. Lancet 2008;371:200-10.

[2] Tschudin S, Bertea PC, Zemp E. Prevalence and predictors of premenstrual syndrome and premenstrual dysphoric disorder in a population-based sample. Arch. Womens Mental Health.2010; 13:485-94. 
[3] Baart ILMA. Bestaat het premenstruele syndroom? (Does the premenstrual syndrome exist?). Huisarts Wet. 1991;34:466-71.

[4] Wittchen HU, Becker E, Lieb R, Krause P. Prevalence, incidence and stability of premenstrual dysphoric disorder in the community. Psychol. Med. 2002;32:119-32.

[5] Halbreich U, Borenstein J, Pearlstein T, Kahn LS. The prevalence, impairment, impact, and burden of premenstrual dysphoric disorder [PMS/PMDD]. Psychoneuroendocrinology 2003;28 Suppl 3:1-23.

[6] Yonkers KA, McCunn KL. Comorbidity of premenstrual syndrome and premenstrual dysphoric disorder with other psychiatric conditions. In: O'Brien PMS, Rapkin AJ, Schmidt PJ, editors. The Premenstrual Syndromes: PMS and PMDD. London: Informa UK Ltd, Taylor and Francis Group, LLC; 2007. p. 49-54.

[7] Kolthoff F, Labots-Vogelesang M, Lagro-Janssen T. Het premenstrueel syndroom. Prevalentie en kenmerken van patiënten in een huisartsenpraktijk. (The premenstrual syndrome. Prevalence and characteristics of patients in a family practice). Huisarts Wet. 2005;48:109-12.

[8] Potter J, Bouyer J, Trussell J, Moreau C. Premenstrual syndrome prevalence and fluctuation over time: results from a French population-based survey. J Womens Health 2009;18:31-9.

[9] Freeman EW. Therapeutic management of premenstrual syndrome. Expert Opin Pharmacother. 2010. 11:2879-89.

[10] Yonkers KA, Simoni MK. Premenstrual disorders. Am J Obstet Gynecol. 2018;218:68-74.

[11] Marván ML, Cortés-Iniestra S. Women's beliefs about the prevalence of premenstrual syndrome and biases in recall of premenstrual changes. Health Psychol. 2001;20:276-80.

[12] Siegel J. Premenstrual syndrome: Psychiatric, physiological, and psychosocial perspectives. Health Soc. Work 1987;12:284-89.

[13] Kadian S, O'Brien PMS. Classification of premenstrual disorders as proposed by the International Society for Premenstrual Disorders. Menopause Int. 2012;18:43-47.

[14] Ward K, Gott M, Hoare K. Participants' views of telephone interviews within a grounded theory study. J. Adv. Nurs. 2015;71:2775-85.

[15] Tong A, Sainsbury P, Craig J. Consolidated criteria for reporting qualitative research (COREQ): a 32item checklist for interviews and focus groups. Int. J. Qual. Health Care 2007;19:349-57.

[16] Asbring P. Chronic illness - a disruption in life: identity-transformation among women with chronic fatigue syndrome and fibromyalgia. J. Adv. Nurs. 2001;34:312-9.

[17] Kelly MP, Field D. Medical sociology, chronic illness and the body. Social. Health Illn. 1996;18:241-57. 
[18] King M, Ussher JM, Perz J. (2014) Representations of PMS and

Premenstrual Women in Men's Accounts: An Analysis of Online Posts from PMSBuddy.com, Women's. Reproductive Health 2014;1: 3-20

[19] Kaiser G, Kues JN, Kleinstäuber M, Andersson G, Weise C. Methods for coping with premenstrual change: Development and validation of the German Premenstrual Change Coping Inventory. Women Health. 2018;58:1062-79.

[20] Roth S, Cohen LJ. Approach, avoidance, and coping with stress. Am. Psychol. 1986;41:813-9.

[21] Ko CH, Yen CF, Long CY, Chen CS, Huang TH, Yen JY. The Association Between Premenstrual Dysphoric Disorder and Internet Use Disorder. Women Health 2014;54:245-61.

[22] Ussher JM, Perz J. PMS as a process of negotiation: women's experience and management of premenstrual distress. Psychol Health. 2013;28:909-27.

[23] Engel GL. From biomedical to biopsychosocial. Being scientific in the human domain. Psychosomatics 1997;38:521-8.

\section{Supplementary Files}

This is a list of supplementary files associated with this preprint. Click to download.

- Table1.pdf

- Table2.pdf

- Table3.pdf

- Box1InterviewGuide.pdf 